\title{
Tratamiento didáctico de aspectos culturales en interpretación
}

\author{
Agustín Darias-Marrero ${ }^{1}$ \\ Universidad de Las Palmas de Gran Canaria
}

\begin{abstract}
This paper deals with the importance of cultural and intercultural elements in the training of interpreters and translators at university. To overcome the pitfalls these elements pose for students, even those with a sound linguistic basis have to use extralinguistic skills. Thus, this paper reviews the national and European legal framework on cultural competence, before considering a teaching proposal. This teaching approach is based on the use of information and communication technology (ICT) in the teaching and learning of liaison interpreting, and promotes the acquisition of cultural and intercultural competences.
\end{abstract}

Key words: interpretation, interpretation training, culture, cultural diversity, ICT.

\begin{abstract}
En este trabajo, se revisa brevemente el marco normativo que regula en la actualidad la competencia cultural de traductores e intérpretes, y la conceptualización de la cultura. Ya en el campo de la didáctica de la interpretación, se reflexiona sobre la competencia cultural y el tratamiento de los elementos con un peso cultural específico en la interpretación. Por último, antes de enunciar unas breves conclusiones, se presentan unas actividades didácticas estructuradas en torno a situaciones de interpretación, que pretenden desarrollar, además de las aptitudes propias de la interpretación, la competencia cultural e intercultural de los
\end{abstract}

1 Corresponding author - Universidad de Las Palmas de Gran Canaria, Departamento de Filología Moderna, Gran Canaria (Spain). Email: agustin.darias@ulpgc.es 
alumnos, al mismo tiempo que se apoya en el uso de las tecnologías de la información y la comunicación (TIC) para favorecer la enseñanza-aprendizaje móvil y autónomo de la interpretación.

Palabras clave: interpretación, didáctica de la interpretación, cultura, diversidad cultural, TIC.

\section{Introducción}

En el marco de la formación universitaria de traductores e intérpretes, los estudiantes deben enfrentarse a incógnitas culturales e interculturales que les plantean problemas, fundamentalmente, de comprensión y de traducción de los discursos originales. Para superarlos, incluso en el caso de aquellos alumnos que dispongan de una base lingüística sólida, se debe utilizar continuamente otras competencias que les permitan concienciarse y adquirir más conocimientos sobre las culturas vinculadas a sus lenguas de trabajo y sobre la interculturalidad inherente al acto comunicativo mediado.

El Libro Blanco del Título de Grado en Traducción e Interpretación, elaborado por la Agencia Nacional de Evaluación de la Calidad y Acreditación (ANECA) en 2004, establece una serie de recomendaciones para los estudios de traducción e interpretación en España. Según esta publicación, los perfiles profesionales de egreso de los estudios de Grado en Traducción e Interpretación se caracterizan, entre otros aspectos, por una serie de competencias, de las cuales algunas mencionan una relación explícita con la cultura, que son las que nos interesan aquí y que se consideran aisladamente a efectos de la exposición, ya que somos conscientes de que toda consideración aislada de la dimensión cultural resultaría artificial. Se podría resumir brevemente esas competencias como la capacidad de poseer interés y conocimientos de las lenguas y culturas A, B y C. En el caso del perfil profesional de intérprete de enlace, se añaden sólidos conocimientos de cultura general, así como la necesidad de conocer los contextos socioculturales y las necesidades comunicativas de las partes implicadas en la situación comunicativa sin tomar partido.

Del mismo modo, el Marco común europeo de referencia para las lenguas (MCER) (2002, p. 99-102) considera, entre las competencias generales del usuario o aprendiz de una lengua extranjera, todos aquellos aspectos que conforman los conocimientos sobre el mundo (características geográficas, medioambientales, demográficas, económicas, políticas, administrativas de los países cuya lengua se estudia), los saberes socioculturales (que pueden ir desde la vida cotidiana hasta las relaciones personales, pasando por aspectos como el lenguaje no verbal, el humor o las convenciones sociales, entre otros valores, creencias y comportamientos) y la conciencia intercultural que permite establecer, a partir de los conocimientos, relaciones entre 
el «mundo de origen» y el «mundo de la comunidad objeto de estudio» (MCER, 2002, p. 101), y también generar una toma de conciencia acerca de cómo aparece la comunidad en cuestión desde la perspectiva de los demás, con frecuencia en forma de estereotipos nacionales, que incluye igualmente las diferencias regionales y sociales en ambos mundos.

Aparte de los conocimientos y la conciencia intercultural, el MCER (2002, p. 102-103) registra destrezas y habilidades interculturales, que son:

- La capacidad de relacionar la cultura de origen y la cultura extranjera.

- La sensibilidad cultural y la capacidad de identificar y utilizar una variedad de estrategias para establecer contacto con personas de otras culturas.

- La capacidad de cumplir el papel de intermediario cultural entre la cultura propia y la ajena, y de abordar con eficacia malentendidos interculturales y situaciones conflictivas.

- La capacidad de superar relaciones estereotipadas.

A partir de la revisión anterior de los conocimientos y competencias recomendados por el marco normativo en los ámbitos nacional y europeo, se puede observar el papel relevante concedido a la cultura y a la diversidad cultural en la formación en traducción e interpretación. Asimismo, resulta evidente que las destrezas interculturales mencionadas antes se ponen realmente en práctica en cualquier situación comunicativa de interpretación, en la que se emplean estrategias comunicativas para poner en contacto a interlocutores de culturas distintas, con lo cual se está ejerciendo el papel de intermediario cultural capaz de ir más allá de los estereotipos y de superar posibles conflictos interculturales.

Entonces, a fin de compensar las posibles carencias culturales e interculturales de los estudiantes, parece adecuado intentar despertar su interés por las culturas y sensibilizarles acerca de la diversidad cultural, además de incrementar sus conocimientos culturales en general. Mejorarían, así, los conocimientos y la capacitación de intérpretes hábiles para desenvolverse en contextos marcados socioculturalmente conservando una actitud respetuosa y neutra hacia los interlocutores implicados. Todo ello constituye precisamente los objetivos de la propuesta didáctica que se presenta en el último apartado y que pretende, mediante la recreación de situaciones de interpretación prototípicas, elevar la competencia cultural e intercultural de los estudiantes.

Con tal fin, parece necesario centrarse a efectos metodológicos y analíticos en el propio concepto de cultura desde el punto de vista teórico, para luego definir las competencias cultural e intercultural. Por último, se tratará cómo inciden los aspectos culturales en la interpretación por oposición a la traducción y se aportarán algunas 
actividades didácticas cuyo objetivo es integrar los aspectos mencionados en la formación en interpretación.

\section{Conceptos de cultura y competencias cultural e intercultural}

\subsection{Conceptualización de cultura}

Dado que el concepto de cultura impregna la reflexión en las ciencias sociales, la traductología no puede sustraerse al signo de los tiempos (Marín Hernández, 2005, p. 74), máxime cuando la traducción y la interpretación como procesos comunicativos interculturales ponen de manifiesto las diferencias culturales. Ahora bien, dadas las numerosas y variadas acepciones que se otorgan al término cultura, hemos revisado algunas definiciones partiendo del campo de la antropología (Tylor, 1871; Kroeber, 1948; Goodenough, 1964; Göhring, 1978; Plog y Bates, 1980; Williams, 1983), que cuenta con «una larga tradición teórica en este terreno» (Marín Hernández, 2005, p. 75), y de la sociología (Rocher, 1969), hasta llegar al de la traductología (Vermeer, 1986, p. 178 y 1992, p. 32; Nord, 1997, p. 24 y 2008; Witte, 2008, p. 65), cuya conceptualización, a la postre, se apoya en los avances de las ciencias humanas anteriormente mencionadas.

A partir de las definiciones consultadas, resulta más apropiado que hablar de cultura, hacerlo de «[...] la pluralité des cultures, par opposition à la Culture (en y mettant la majuscule d'une valorisation emphatique et traditionnaliste, voire élitiste)» (Ladmiral, 1998, p. 16), «puesto que no existe una cultura sino diversas culturas que son sólo significantes para los miembros que forman parte de cada una de ellas y se identifican con la suya en contra de las de los otros» (Martínez Sahuquillo, 1997, p. 186). Estas pueden caracterizarse como:

- Las culturas son todo lo que se aprende (Kroeber, 1948, p. 17; Plog y Bates, 1980, p. 19).

- Están constituidas por modos de percepción e interpretación de la realidad. No consisten solamente en conductas manifiestas, sino también en ideas, creencias, valores que subyacen a esas conductas (Plog y Bates, 1980, p. 19).

- Son simbólicas. Los sistemas simbólicos humanos se construyen sobre elementos convencionales que se combinan para producir mensajes complejos y abstractos, especialmente en el más avanzado de ellos, el lenguaje, incluido el no verbal.

- Son un todo estructurado (Guy Rocher, 1969, p. 88), sistemas integrados en los que existe una interrelación entre creencias, valores, costumbres e instituciones.

- Son compartidas por los individuos como integrantes de un grupo que se distingue de otros grupos (Kottak, 1994, p. 38) en función de criterios de 
generación, posición socioeconómica, ocupación, origen, género, religión, etc.

- Por lo tanto, no son monolíticas, sino intrínsecamente heterogéneas y plurales (Williams, 1983, p. 89).

- Son dispositivos de adaptación al medio natural y social, aunque existen también fenómenos contrarios a la adaptación.

- Son cambiantes y dinámicas (Williams, 1983, p. 89), a pesar de resistencias ante los cambios, sobre todo, en los tiempos actuales globalizados y con las distancias físicas prácticamente anuladas por los medios de comunicación de masas y las TIC.

Además de los rasgos mencionados que no pretenden ser exhaustivos y teniendo en cuenta que la propia reflexión teórica sobre el concepto evoluciona (Koskinen, 2004, p. 150), consideramos a continuación otros tres aspectos relevantes en la conceptualización de las culturas. En primer lugar y según los estudios de antropología, sociología y de comunicación intercultural, se pueden contemplar varios niveles de cultura: nacional, internacional, subcultural (Kottak, 1994); o bien, paraculturas y diaculturas (Vermeer, 1992, p. 32), respectivamente como lenguaestado-nación y grupos profesionales y de intereses de una paracultura (Witte, 2000, p. 56); se puede hablar también de idiocultura, para referirse a la cultura de un individuo (Witte, 2008, p. 60). En cualquier caso, como categoría teórico-metodológica, hay que especificar un nivel determinado de análisis cuando se va a trabajar con las culturas.

En segundo lugar, sea cual sea el marco de análisis escogido, no se tiene por qué hacer coincidir el concepto de cultura con otras nociones: «In research and curricula, cultural and linguistic boundaries are perceived as identical, and coinciding with national borders» (Koskinen, 2004, p. 144). Con frecuencia, la cultura se confunde con la lengua, categoría lingüística definida en términos generales como el lenguaje empleado por una comunidad de hablantes, de los que no todos deben obligatoriamente sentirse identificados con una única cultura concreta. También suele confundirse con la noción de estado-nación, estructura político-administrativa, gestada históricamente y delimitada territorialmente, que puede albergar $y$, de hecho, alberga varias culturas.

En tercer lugar, cabe destacar que las culturas pueden abordarse a partir de una escala que oscila entre lo universal y lo particular. En general, todos los humanos compartimos una serie de universales, como el amor por nuestros seres más cercanos, familia y amigos, o los hábitos de la comida; generalidades que comparten un determinado grupo de culturas, como el comer sentados a la mesa en las culturas occidentales; y particularidades propias de una tradición cultural, como comer el queso en aperitivo antes de la comida en España y después en Francia; y ello, a pesar 
de que podamos encontrar a alguien de cultura francesa cuya idiocultura le lleve a comer el queso entre comidas y sentado en el suelo delante de su televisor.

Como consecuencia de lo anterior, la traducción y la interpretación en tanto que actividades comunicativas humanas implican la dimensión psicológica individual y, de entre la multiplicidad de factores y relaciones interpersonales que se pueden dar entre los actores de un acto comunicativo mediado por un traductor o un intérprete, los más relevantes son, sobre todo, de índole cognitiva y motivacional (Darias Marrero, 2009, p. 42-43). No obstante, un individuo es representante de la cultura en la que evoluciona y no puede sustraerse al marco sociocultural en el que se forjó su personalidad y que le sirve de referencia para el uso de la infraestructura lingüísticocultural en vigor en su comunidad (Félix Fernández, 2004, p. 36).

\subsection{Competencias cultural e intercultural}

Desde un enfoque didáctico de la traducción y la interpretación, el término cultura suele emplearse en general con dos acepciones. Por una parte, se usa fundamentalmente para designar todo aquello que resulta difícil traducir con palabras, todo aquello que la herramienta lingüística no consigue abarcar, lo que se denomina normalmente en sentido amplio con la etiqueta de problemas de traducción:

Por regla general, la «cultura» se situaba en el punto de mira sólo cuando se observaban «problemas» culturales durante el proceso traslativo, cuando, p. ej., resultaban imprescindibles determinados conocimientos extralingüísticos para entender o reformular un pasaje textual (Witte, 2008, p. 55).

En este sentido, se emplea como si pudiera establecerse una separación tajante entre unidades lingüísticas y unidades culturales de traducción, lo cual se entiende solamente «desde un concepto esencialista y trascendente de la cultura» (Marín Hernández, 2005, p. 80) y se explica, entre otras posibilidades, porque también la cultura se ha definido por oposición a la lengua, «defined by what it was not: culture as anti-language; the cultural paradigm as anti-linguistic» (Koskinen, 2004, p. 150).

Por otra parte, en cuanto al segundo empleo del término cultura, se usa también para referirse a las competencias cultural, en las culturas de partida y de llegada, e intercultural del traductor. Con este significado se referiría al bagaje cultural del traductor, es decir, su cultura general o conocimientos enciclopédicos. Por ejemplo, Hurtado Albir (1999), en su propuesta metodológica examina las competencias necesarias para formar a traductores e intérpretes y analiza la competencia traductora dividiéndola en subcompetencias (Hurtado Albir, 1999, p. 42-44; PACTE, 
2014, p. 88), de las que citamos la definición de la más pertinente para nuestros fines:

Extra-linguistic sub-competence. Predominantly declarative knowledge, both implicit and explicit, about the world in general and special areas. It includes: (1) bicultural knowledge (about the source and target cultures); (2) encyclopaedic knowledge (about the world in general); (3) subject knowledge (in special areas) (PACTE, 2003, p. 58-59).

También Witte define la competencia cultural necesaria para traductores e intérpretes como sigue:

[...] la capacidad de tomar conciencia crítica de lo que se «sabe» de forma inconsciente y de «aprender» conscientemente lo que no se «sabe» de la propia cultura y de otra(s) cultura(s), así como la capacidad de relacionar y contrastar dichas culturas con el fin de poder recibir y producir comportamientos de acuerdo con el objetivo de la comunicación y orientados a la situación comunicativa, con respecto a las necesidades comunicativas de al menos dos actantes de dos culturas distintas, con el fin de hacer posible la comunicación entre estos actantes (Witte, 2005, p. 50).

De forma que la competencia cultural en la cultura propia y en la ajena, así como la competencia intercultural, deben formar parte del perfil de traductores e intérpretes. Además, Witte (2005, p. 50-51) también distingue en el seno de la competencia cultural dos subcompetencias: la competencia en culturas y la competencia entre culturas. Con respecto a la primera, manifiesta que se refiere a las tendencias de percepción y a los patrones de comportamiento, actitudes, valores, etc.; con lo cual, abarca más que los meros conocimientos e informaciones sobre una cultura dada. De manera que su concepción de esta subcompetencia resulta satisfactoria al adaptarse a las definiciones de cultura consideradas antes, por contemplar todos los aspectos tradicionalmente denominados culturales, al igual que aquellos menos observables y más abstractos que configuran todo el patrimonio intangible y comportamental global de una cultura. Sin embargo, parece necesario constatar que, en los estudios traductológicos, el intento de sobrepasar la barrera de los patrones, modelos, prototipos, tendencias, siempre generales y orientativos, se complica verdaderamente, dada la unicidad de cada acto comunicativo. Precisamente, la comunicación como actividad humana remite a individuos, integrados en una sociedad y una cultura, en calidad de protagonistas de la interacción (Darias-Marrero, 2009, p. 42):

[...] las necesidades comunicativas que intenta resolver la traducción se dan siempre en individuos, agrupados o no, cuyas representaciones del mundo - sus conjuntos de estructuras conceptuales o cultura particular coinciden tan sólo parcialmente con la cultura general de su comunidad lingüística (Muñoz Martín, 1999, p. 162). 
La competencia entre culturas, la segunda subcompetencia de Witte, se refiere a la capacidad de formular hipótesis sobre los conocimientos previos que puede tener un interlocutor acerca de otro, así como a la capacidad de estimar cómo dichos conocimientos previos pueden influir sobre el comportamiento global de los interlocutores en la situación de encuentro interlingüe e intercultural. Por lo tanto, la competencia cultural, a grandes rasgos hipotética, se actualizará y adaptará en función de cada situación comunicativa concreta, con el fin de poder anticipar y corregir posibles comportamientos culturalmente inadecuados por parte de los interlocutores o interactantes, según la terminología de Witte, puesto que no se refiere solamente a su comportamiento como hablantes, sino a su comportamiento global.

En consecuencia, si bien las competencias en culturas y entre culturas son básicas para traductores e intérpretes, resultan dinámicas y relativas en función de cada situación comunicativa única. En efecto, siguiendo a Pym (2003, p. 454-455), los traductores e intérpretes por sus conocimientos sobre el propio proceso traslativo cruzan continuamente la supuesta frontera existente entre las culturas, situándose en ese espacio desde el que, gracias a sus conocimientos, no perciben las diferencias culturales como una barrera, sino como algo que se construye a posteriori, después de que han tenido que solventar un punto de contacto intercultural en una traducción o interpretación concretas, mediante la herramienta lingüística y recurriendo también a sus conocimientos extralingüísticos indisociables de los lingüísticos, tal y como hemos avanzado antes. Las competencias cultural e intercultural de traductores e intérpretes engloban, pues, conocimientos teóricos sobre los puntos de contacto intercultural y su incidencia en los procesos de traducción y de interpretación, así como conocimientos sólidos sobre las culturas origen y meta, que les permitan localizar los elementos de la cultura extranjera relevantes en una situación comunicativa concreta y tratarlos en consonancia con las necesidades comunicativas de un público determinado.

\section{3. Diferencias entre traducción e interpretación}

Ahora bien, parece claro que los aspectos culturales se afrontan de diferente manera en la traducción escrita o en la interpretación oral, considerando las condiciones en las que se realiza una u otra actividad traslativa. Dadas las diferencias existentes entre la traducción escrita y la oral, nos limitaremos a resaltar dos condicionantes que marcan especialmente la forma en que se lleva a cabo cada actividad:

- La duración de la actividad. A pesar de los cortísimos plazos que imponen los clientes a los traductores, en general pueden disponer de tiempo suficiente para documentarse sobre el tema y consultar fuentes de información documentales y 
humanas. Por el contrario, la interpretación se realiza en el marco de la situación comunicativa real, por lo que se espera del intérprete que restituya inmediata e improvisadamente el mensaje, incluso el más especializado, al ritmo impuesto por el orador, recurriendo a sus propios conocimientos lingüísticos y extralingüísticos, incluidos los culturales. Para afrontar esta dificultad, los intérpretes profesionales se informan y documentan previamente sobre el tema y el encuentro en el que trabajarán.

- La coincidencia temporal de las operaciones cognitivas encaminadas a la recepción y a la producción del mensaje. En la didáctica de la traducción escrita, se aconseja disociar las fases de recepción y producción del mensaje (García López, 2004). El traductor puede, así, aproximarse al texto original para leerlo, analizarlo, comprenderlo y, después, centrarse en la expresión en la otra lengua del sentido que ya ha captado. Además, puede revisar posteriormente la formulación elegida. Sin embargo, todas estas tareas de recepción y producción del discurso oral coinciden plenamente en la interpretación simultánea, durante la cual el intérprete escucha, analiza y comprende el discurso de origen, al mismo tiempo que formula su propio discurso en lengua meta. Todo ello le exige, entre otras cosas, una enorme concentración para realizar varias operaciones cognitivas simultáneamente. También en la práctica de la interpretación consecutiva, todas estas destrezas se siguen muy de cerca.

A raíz de lo mencionado, parece evidente que los posibles aspectos culturales que surjan a lo largo del proceso traslativo en una situación de traducción o de interpretación no se pueden afrontar igualmente. Así, para la comprensión íntegra de un texto escrito y su posterior traducción, habría bien que contar con unos conocimientos culturales previos, bien que realizar toda una búsqueda documental para desentrañar el sentido de cualquiera de los numerosos aspectos característicos de una cultura revisados brevemente en el primer apartado y que pudieran ser relevantes en una situación comunicativa dada. Cuando se presuponen unos conocimientos culturales compartidos por el emisor y los destinatarios del texto, orientados a provocar un determinado efecto pragmático en éstos últimos, el traductor de textos escritos puede realizar un proceso investigador con el fin de afrontar aquellos aspectos culturales que pudieran representar un obstáculo para la transmisión plena del sentido del texto original.

Del mismo modo, resulta evidente que, en la fase de elaboración del texto meta, el traductor cuenta con tiempo para reflexionar sobre su texto y usa varios recursos con el objetivo de asegurar la transferencia intercultural. La libertad del traductor de un texto escrito se plasma en la elección de los procedimientos de los que dispone, entre otros posibles, conservar los elementos en lengua original, explicarlos en lengua meta o recurrir a signos tipográficos, como la cursiva o las comillas, para salvar con mayor o menor éxito los escollos representados por la transmisión intercultural de aspectos 
como, por ejemplo, el humor o los juegos de palabras.

Asimismo, otra técnica disponible en traducción escrita para salvar los problemas que nos ocupan es la nota del traductor. Incluso si el empleo de las notas del traductor se considera a menudo en el mundo profesional como la confesión de una derrota por parte del traductor, según Corredor Plaja (2011, p. 151), la inclusión de notas en el texto de la traducción debería depender del criterio del propio traductor que ha optado por utilizarlas para transmitir un contenido que difícilmente hubiera resultado aceptable en la cultura meta $y$, en ese caso, el traductor ya no presupone a los destinatarios de su traducción los conocimientos suficientes para comprender determinados aspectos culturales.

El traductor, como mediador, emplea todos los recursos a su alcance para exteriorizar la falta de correspondencia exacta entre lenguas y culturas, y compartirla con el lector (Corredor Plaja, 2011, p. 163). Por consiguiente, parece obvio que los traductores cuentan con condiciones distintas a las de los intérpretes para lograr la transmisión intercultural e interlingüística. Por ejemplo, Newmark (1999, p. 145-146) propone contrastar las culturas en juego e identificar sus diferencias con respecto a una serie de factores contextuales. Tras su análisis, postula hasta doce procedimientos distintos, cuya descripción no tiene cabida en el marco de este estudio, para el tratamiento del elemento cultural en cuestión. En cualquier caso, parece claro que «hay un sinnúmero de soluciones según el tipo de textos» (Lvóvskaya, 1997, p. 49-50).

\section{Tratamiento didáctico de aspectos culturales en formación en interpretación}

\subsection{Incidencia de los aspectos culturales en interpretación}

No obstante, la falta de correspondencia exacta en la forma de organizar la realidad en distintas culturas y lenguas, suscita problemas de cara al trasvase intercultural e interlingüístico, lo que implica que se produzcan ganancias y pérdidas durante el proceso traductor. Si esto es cierto para la traducción escrita, lo es aún más para la interpretación, en la que puede haber pérdidas de información del discurso original. Además, el tipo de análisis y algunos de los procedimientos mencionados antes, no se pueden emplear teniendo en cuenta que, en las condiciones antedichas de realización de la actividad, resulta imposible efectuar un análisis tan detallado del discurso original en situación de interpretación. Ayudado por el sentido extralingüístico que se desprende de la propia situación comunicativa, el discurso interpretado resultará aceptable en la cultura meta, puesto que respeta en la medida de lo posible los motivos y los objetivos del orador, y las convenciones verbales de la cultura meta, aunque puede que no se adapte perfectamente desde el punto de vista 
conceptual y formal a la nueva situación comunicativa en la cultura de llegada.

El intérprete no dispone de todas las posibilidades mencionadas sucintamente para la traducción $y$, en el mejor de los casos, ante este tipo de elementos con una determinada carga cultural, debe contentarse con introducir en su discurso una explicación rápida, concisa y acertada para cumplir con éxito inmediato su función comunicativa. Si el ritmo del orador se lo permite, incluso podría conservar el término original en cuestión seguido de su traducción o explicación. Pero, en cualquier caso, el intérprete tendrá que actuar con celeridad para no perder el hilo conductor del discurso original y de la forma más neutra posible para no atraer demasiado la atención de sus oyentes sobre su propia tarea ya que, al igual que en las notas del traductor, las explicaciones harán que emerja su subjetividad en el discurso interpretado.

Teniendo en cuenta, entonces, las diferencias expuestas entre los procesos traslativos escritos y orales, desde el punto de vista didáctico, a la dificultad mencionada arriba vendría a añadirse el frecuentemente escaso conocimiento del mundo de los estudiantes de interpretación (Kurz, 2003, p. 59) que, por su propia edad no pueden contar todavía con un extenso bagaje cultural. Asimismo, hay que considerar la inferioridad de conocimientos temáticos en comparación con los demás interlocutores del acto comunicativo, que poseen, sin duda, la formación apropiada para captar todos los matices del mensaje. Además, una gran parte de los temas tratados en interpretación pertenecen a la esfera de los conocimientos especializados o a parcelas de conocimiento muy concretas y punteras, lo que no puede sino contribuir a dificultar aún más el tratamiento del mensaje oral incluso para los intérpretes experimentados. De ahí, que resulte pertinente reforzar las competencias cultural e intercultural en la formación en interpretación.

Ahora bien, considerando que cada acto comunicativo es único, resultaría extremadamente complejo establecer todos los posibles aspectos culturales que podrían aparecer en el transcurso de una interpretación concreta puesto que, potencialmente, podría ser cualquiera, aunque los aspectos más relevantes estarían, sin duda alguna, relacionados con las culturas involucradas, la situación comunicativa y el tema tratado. Sin ninguna pretensión de exhaustividad, algunas de las dificultades que se plantean con frecuencia en interpretación a causa de la disimetría entre lenguas y culturas se refieren a nombres propios, topónimos, siglas o acrónimos, denominaciones de instituciones y las relativas a la organización políticoadministrativa de los países en cuestión, convenciones sociales y rasgos de comportamiento no verbal. Así, la competencia instrumental, entendida como conocimiento sobre el uso de fuentes de documentación y el uso de las TIC (PACTE, 2003, p. 59), se revela como una de las mejores herramientas con las que cuenta el intérprete para documentar y preparar previamente el encuentro en el que mediará, 
con lo cual habrá de anticipar, en la medida de lo posible, las eventuales dificultades, por supuesto lingüísticas, pero también las que surjan del estrecho contacto entre culturas en una situación de interpretación determinada. Por lo tanto, en la didáctica de la interpretación habrá que tener en cuenta todos aquellos conocimientos que pueden incidir en el proceso de la interpretación y en las prestaciones de los estudiantes. De forma que éstos deben recurrir a toda su cultura general para mejorar sus resultados en interpretación y los docentes debemos favorecer, por todos los medios, la mejora de sus conocimientos culturales y su sensibilización hacia la diversidad cultural.

Dicho esto, las diferencias culturales más o menos pertinentes en una situación comunicativa incidirán de forma distinta en función de los contextos profesionales en los que intervenga el intérprete. En ese sentido, aunque su caracterización detallada excede el marco de este estudio, habría que considerar las diversas modalidades de interpretación comúnmente admitidas (de conferencias, ante los tribunales, social o de enlace) que se relacionan con los eventos comunicativos y situaciones sociales en los que el intérprete realiza su trabajo (Bowen et al., 1995, p. 245). En la interpretación de conferencias, aunque cada interlocutor tenga su propia identidad y su propia cultura, se impone la noción de diacultura internacional (Pöchhacker, 2003, p. 119) que permite atenuar las diferencias, puesto que los participantes en uno de estos eventos internacionales comparten de alguna manera la cultura común de las conferencias o congresos internacionales especializados. Las modalidades que presentan diferencias interculturales más evidentes son la interpretación social, en la que aquéllas se acompañan normalmente de diferencias de nivel social o de posición de poder, y la interpretación de enlace (piénsese, a modo de ejemplo, en un encuentro comercial entre un empresario francés y otro japonés), en las que se da un continuo trasvase entre las convenciones sociales, las creencias y las representaciones del mundo de los interlocutores. Además, en estos contextos socioprofesionales, se suele interpretar mayormente de forma bilateral en ambos sentidos de la combinación lingüística del intérprete. A grandes rasgos, la interpretación bilateral se define como:

La mediación oral que se realiza por un solo intérprete, en las dos direcciones, e inmediatamente después de cada una de las intervenciones de los interlocutores presentes en la situación comunicativa. El contacto directo, la bidireccionalidad y la dimensión interpersonal de la interacción cara a cara constituyen sus rasgos más distintivos (Collados Aís y Fernández Sánchez, 2001, p. 48).

\subsection{Actividades didácticas de sensibilización}


Por las razones expuestas hasta ahora, Collados Aís y Fernández Sánchez (2001, p. 123-124) favorecen la concienciación intercultural en la formación en interpretación bilateral y proponen algunos ejercicios específicos relativos a la interculturalidad, al mismo tiempo que reconocen que en interpretación hay pocos ejercicios dedicados específicamente a este aspecto. Algunas actividades, entre otras posibles, serían:

- Presentación de videos y juegos de roles sobre situaciones comunicativas de interpretación, para tratar, entre otros posibles, aspectos profesionales, teóricos e interculturales.

- Práctica y adquisición de estrategias comunicativas conversacionales, orientadas a trabajar con expresiones convencionales y fórmulas codificadas relativas a la dinámica del intercambio comunicativo (saludarse, despedirse, reglas de cortesía, tú/usted, solicitar más información, rechazar un argumento).

- Ejercicios de precisión léxica, encaminados a valorar y clasificar series de sinónimos en función de su empleo y connotaciones emotivas, y elaboración de glosarios léxicos para comparar el empleo de los términos en las distintas culturas (Collados Aís, 2004).

En la misma línea, se intenta sensibilizar a los estudiantes acerca de la interculturalidad inherente al acto mediado en el marco de la formación universitaria impartida en las asignaturas de interpretación de cuarto curso del Grado en Traducción e Interpretación, orientadas a alumnos cuyo perfil lingüístico se puede caracterizar, en términos generales, por tener el francés como segunda lengua de trabajo y, en consecuencia, escasos y desiguales conocimientos sobre la lengua y las culturas francófonas.

A continuación se exponen algunos ejemplos de actividades didácticas de sensibilización realizadas a lo largo de esta formación generalista en interpretación, cuyo objetivo es la concienciación de los estudiantes sobre cómo pueden incidir aspectos culturales relevantes mientras se interpreta en situaciones de interpretación concretas. En estos ejercicios los estudiantes escuchan, primero, frases extraídas de la prensa y, luego, fragmentos de discursos sacados de la práctica profesional real, que sirven de material didáctico y han sido elegidos por la presencia de numerosos aspectos culturales, y se les pide que reformulen su sentido con el fin de que tomen conciencia de la dificultad implícita en el tratamiento de los elementos en cuestión.

Las frases y fragmentos de discurso propuestos pueden contener, por ejemplo, cualquier tipo de denominación de referencias culturales, nombres propios de personas, objetos o lugares, cualquier elemento cuya apelación dificulta su escucha (Gile, 1984) y, sobre todo si es desconocida, casi imposibilita el punto de partida para el procesamiento de la información en interpretación, es decir, la comprensión oral, máxime considerando que la concentración del intérprete se reparte para realizar 
varias tareas cognitivas simultáneamente como explica Gile en su modelo de esfuerzos $(1985,1988)$. Por lo consiguiente, esta dificultad puede conllevar cambios o pérdidas de información en el discurso interpretado.

Pero, aunque los estudiantes los escuchen correctamente y logren calcar sus referentes fonéticamente en ocasiones, deberían conocer esas referencias culturales para poder comprender lo designado en la realidad a la que se alude y captar plenamente el sentido del mensaje, sin limitarse a una mera transcodificación o traducción literal, es decir, «à un transfert direct d'unités linguistiques de la langue source à la langue cible par correspondances préétablies» (Gile, 2004). En efecto, la transcodificación de términos $\mathrm{y}$, por ejemplo, de nombres propios, tendría que completarse con un trabajo de comprensión del discurso original para poder producir un discurso interpretado (Gile, 2004) adaptado a la situación comunicativa y a los interlocutores en juego.

De igual modo, se presenta a los estudiantes fragmentos textuales contextualizados en determinadas situaciones de interpretación bilateral, que contienen aspectos culturales relativos a la distinta forma de organizar la realidad en diferentes culturas. Por ejemplo, se usan textos orales que mencionen instituciones o divisiones administrativas de los países en cuestión, como los «DOM» franceses en un contexto de diálogo entre autoridades canarias y martiniqueñas, o una «wilaya» en un encuentro canario-marroquí. En función de la relevancia de estos elementos en la situación concreta, su tratamiento puede exigir una explicitación (Gumul, 2008) por parte del intérprete con el fin de proporcionar un mejor servicio a sus oyentes.

Asimismo, se pretende concienciar a los alumnos acerca de las convenciones sociales y rasgos de comportamiento no verbal marcados culturalmente. Siempre a título ilustrativo, se les puede presentar una situación de interpretación bilateral en la que los interlocutores, en este caso, organizadores de una regata, no consigan entenderse a causa de distintas concepciones de aspectos culturales, como el concepto de «mediodía/midi», en cuanto a la franja horaria y la correspondiente comida se refiere (almuerzo entre 14-15 horas y «déjeuner à midi»), para hablantes españoles y franceses.

A pesar de lo dicho, los conocimientos culturales inciden también de manera positiva en el proceso de la interpretación. De hecho, con frecuencia se destaca la importancia de una sólida cultura general para los intérpretes y ya hemos mencionado la competencia extralingüística que abarca todo tipo de conocimientos: culturales, enciclopédicos y temáticos. Nunca se sabe en qué momento de una interpretación una determinada información puede resultar esencial para poder transmitir el mensaje.

Por ejemplo, durante la realización de estas actividades de sensibilización sobre 
aspectos culturales, un estudiante interpreta erróneamente el nombre francés del oficio de «buraliste» como «oficinista», empleado en una oficina, en un «bureau». Se le explica que un «buraliste» trabaja en un «bureau de tabac», que es un estanco, un quiosco. El estudiante en cuestión comprende entonces de qué se trata pero, considerando la inmediatez de la realización de la interpretación y sin poder usar un diccionario, sigue sin encontrar cómo se denomina esa profesión en español, hasta que le sirve de ayuda una realización cultural, la película La estanquera de Vallecas, con lo que, recurriendo a sus conocimientos culturales, logra establecer la equivalencia léxica entre «buraliste» $y$ «estanquero».

Haciéndonos eco de lo anterior, se podría avanzar, entonces, que uno de los objetivos de la formación en interpretación bilateral debería ser que los estudiantes se concienciaran de su función intercultural. En consecuencia, la diversidad cultural debería considerarse un tema transversal en todas las actividades orientadas a la enseñanza-aprendizaje de la interpretación. De ahí, la necesidad didáctica de integrar la dimensión cultural e intercultural en la formación de intérpretes.

\subsection{Proyectos didácticos}

Las actividades que se presentan a continuación surgen de la constatación anterior y se apoyan sobre proyectos realizados previamente. El primero es un proyecto de innovación educativa (PIE) de la ULPGC Ilevado a cabo entre 2011 y 2012, denominado «Grabación, edición y creación de recursos SCORM para el aprendizaje móvil de lenguas extranjeras en un contexto universitario» ${ }^{2}$, y cuyo objetivo principal consistía en crear, grabar y editar documentos multimedia y objetos didácticos (learning objects) sujetos a la norma SCORM (Shareable Content Object Reference Model), para la enseñanza-aprendizaje de lenguas extranjeras en los niveles A1, A2, B1 y B2 definidos en el MCER. En ese proyecto se empezó a orientar el uso de las TIC al campo de la formación en interpretación, concretamente, de la interpretación bilateral, como herramienta complementaria a la enseñanza presencial.

Un segundo proyecto se enmarca en la convocatoria de proyectos Prometeo y fue realizado entre 2012 y 2013 con financiación de la ULPGC. Gracias a este proyecto se grabaron vídeos con situaciones simuladas para la práctica de la interpretación bilateral, en las que se intentó incluir convenciones sociales y patrones comportamentales marcados culturalmente.

Debido a la necesidad de alojar los recursos didácticos en una plataforma para que

https://www2.ulpgc.es/index.php?pagina=vp\&ver=proy_inno_edu_2011 y

https://www2.ulpgc.es/index.php?pagina=vp\&ver=2011_pie03 [acceso: 26-09-16]. 
estuvieran disponibles en cualquier momento y lugar, se lleva a cabo otro PIE en la convocatoria de $2013^{3}$ para el «Desarrollo de la página Web Ubilingua para el aprendizaje multilingüe». El sitio $\mathrm{Web}^{5}$ resultante incluye una sección con recursos dedicados específicamente al aprendizaje de la interpretación que irá enriqueciéndose progresivamente.

La evolución de la línea investigadora e innovadora expuesta someramente (DariasMarrero, García Sánchez y Vidal Luengo, 2013), cristaliza la iniciativa de aplicar el mismo método de creación de recursos docentes a la enseñanza-aprendizaje de la interpretación bilateral y se obtiene, así, el actual PIE en curso en la convocatoria de 2015, denominado «Desarrollo de materiales didácticos para la interpretación de enlace y lenguas con fines específicos» ${ }^{6}$. Una de las posibles innovaciones de la propuesta didáctica que incluye reside en el empleo de las TIC en el ámbito de la formación en interpretación, siguiendo la misma tendencia de otras herramientas virtuales existentes que contienen recursos didácticos para la interpretación como, por ejemplo, el repositorio de discursos de la Comisión Europea?.

Por un lado, la propuesta didáctica planteada pretende, mediante el formato informático de los objetos didácticos SCORM, fomentar la enseñanza y el aprendizaje electrónicos y móviles de la interpretación, ya que facilita la difusión de los materiales didácticos en cualquier plataforma virtual de aprendizaje, con lo cual estarían disponibles en cualquier momento y lugar. Por otro lado, gracias a la práctica de las destrezas orales y de la interpretación bilateral, con otras actividades guiadas y programadas por el docente, se invita al alumno a participar en su aprendizaje activa y autónomamente profundizando en los conocimientos sobre las culturas en cuestión. De esta forma, la utilización de las TIC refuerza la enseñanza presencial, al mismo tiempo que potencia el aprendizaje electrónico, móvil y autónomo.

Los objetos didácticos SCORM presentados sucintamente aquí, pueden integrar, mediante un hilo conductor concreto, situaciones comunicativas de interpretación, por ejemplo, la pérdida del equipaje en un aeropuerto internacional o acudir al colegio de un hijo o al hospital en un país extranjero, con contenidos culturales, es decir, engloban en un único recurso didáctico grabaciones audiovisuales con

3 https://www.ulpgc.es/vp/proyectos-innovacin-educativa-2013-0 [acceso: 26-09-16].

$4 \quad$ https://www2.ulpgc.es/index.php?pagina=vp\&ver=cpie2013_31 [acceso: 26-09-16].

5 http://www.webs.ulpgc.es/webidiomas/ubi/?lang=es [acceso: 27-09-16].

$6 \quad$ https://www.ulpgc.es/search/gss/CPIE2015-31 y https://www2.ulpgc.es/hege/almacen/download/7118/7118256/boulpgc_febrero_2016_5_febrer o.pdf [acceso: 26-09-16].

7 https://webgate.ec.europa.eu/sr/ [acceso: 26-09-16]. 
simulaciones de situaciones comunicativas para la interpretación de enlace y actividades complementarias orientadas, entre otras posibles aplicaciones, a la mejora de los conocimientos sobre las culturas en juego. Con lo cual, esta solución tecnológica favorece el trabajo simultáneo de distintas destrezas y las competencias que se pretende desarrollar son las que se ponen en práctica en la interpretación bilateral y la traducción a la vista, así como las competencias cultural e intercultural de los estudiantes.

Por lo tanto, los recursos que se han elaborado hasta ahora están organizados en función de las competencias mencionadas arriba y, a título ilustrativo, se estructuran en torno a:

- Una serie de actividades previas e introductorias a la interpretación, como pueden ser todas aquellas que se orientan a la adquisición de las destrezas necesarias para realizar una interpretación (Darias-Marrero y Pérez-Luzardo Díaz, 2003, p. 155-156). A modo de ilustración, podría tratarse de actividades sobre el léxico relativo a una situación determinada de interpretación bilateral o la práctica exhaustiva de conversaciones interlingüísticas con el fin de lograr un cierto grado de automatismo en el uso de las formulaciones hasta cierto punto codificadas que se emplean en este tipo de encuentros.

- La interpretación bilateral propiamente dicha de una situación comunicativa simulada, grabada en vídeo. Las situaciones citadas antes sirven de ejemplo y se orientan a la puesta en práctica de todas las competencias mencionadas. En cualquier caso, se pueden preparar otros encuentros que pongan en escena a una persona que no habla la lengua del país donde se encuentra y que debe acceder a los servicios públicos de ese país, y a un representante de los mismos, ya sean servicios administrativos, educativos, sanitarios o sociales. Un ejemplo de este tipo de situaciones interlingües podría ser la de una mujer extranjera víctima de violencia de género que tenga que presentar una denuncia en una comisaría de policía. Son situaciones comunicativas cuyo desarrollo entraña a menudo un determinado protocolo, un cierto grado de redundancia de una situación a otra, de forma que se puedan aproximar tales simulaciones concretas a situaciones arquetípicas de la interpretación de enlace. En la medida de lo posible, se trata de que el aprendiz sea capaz de conocer, primero, y de anticipar, después, los factores más relevantes que configuran y pueden suscitar dificultades, en términos generales, en una situación de interpretación, y que pueden igualmente guiar su preparación y su documentación previas ante una situación de interpretación concreta.

- La traducción a la vista de documentos escritos relativos a la situación. Se considera oportuno incluir esta actividad puesto que potencia la agilidad mental y la capacidad para traducir sin preparación previa un mensaje de una lengua reformulándolo en un texto coherente en otra lengua y que sea aceptable para la cultura meta. La práctica de estas destrezas resulta particularmente pertinente en 
las situaciones de interpretación bilateral en las que, con frecuencia, el grado de formalismo antes citado hace que el intérprete deba enfrentarse a la traducción a la vista de documentos estandardizados requeridos por el tipo de situación. Los documentos pueden ser formularios administrativos en los que se consignan, entre otros, los datos personales, pliegos en los que se informa sobre sus derechos a un detenido, denuncias policiales, diagnósticos médicos.

- Por último, se incluirían también actividades sobre contenidos culturales, seleccionados en función de una relación lógica con la situación simulada. Los recursos SCORM están dotados de un hilo conductor, a modo de unidades didácticas, que facilita la incorporación de elementos culturales orientados a la mejora de los conocimientos culturales de los estudiantes y les sensibilice sobre la diversidad cultural, o sea, que desarrolle su competencia cultural e intercultural. A modo de ejemplo, la simulación del encuentro entre la madre de un alumno y el director del colegio en el extranjero, permite que el estudiante se familiarice con formularios administrativos como el de matrícula escolar mediante la traducción a la vista de este tipo de documentos, al mismo tiempo que permite introducir conocimientos más amplios como la comparación del sistema educativo español y francés. Del mismo modo, se pueden añadir todo tipo de soportes didácticos que ilustren aspectos culturales e interculturales, como por ejemplo presentaciones PowerPoint o direcciones URL que lleven directamente a contenidos de libre acceso en Internet, vídeos en YouTube o artículos de Wikipedia, que ilustren todo tipo de aspectos relevantes sobre el tema y las culturas en cuestión. Con respecto a los contenidos culturales e interculturales recogidos en la normativa en vigor, los objetos didácticos SCORM pueden contener distintos recursos multimedia que permiten profundizar a voluntad en los contenidos mencionados, encauzando asimismo la búsqueda documental del estudiante.

\section{Conclusiones}

Entre otras muchas posibilidades, esta propuesta didáctica permitiría tipificar y practicar con situaciones y estrategias comunicativas de interpretación, así como con determinados tipos de textos que se pueden asociar a ellas. De forma que, si bien es imposible, dadas la naturaleza improvisada de la interpretación y de la comunicación como actividad humana, prever todas las potenciales dificultades debidas a la problemática intercultural, se anticipen los posibles imprevistos y, en consecuencia, se minimicen los riesgos.

Por otro lado, en cuanto al tratamiento de los posibles escollos interculturales, con todos los medios técnicos a nuestro alcance hoy en día, se pueden concebir numerosos tipos de actividades distintas orientadas a la mejora de la competencia cultural y de la concienciación sobre la diversidad cultural de los alumnos de 
interpretación, con el fin de que sean capaces de superarlos y lograr su función mediadora con éxito.

Dada la dimensión intercultural de la interpretación como actividad que posibilita la comunicación entre individuos de distintas culturas, los aspectos relativos a éstas deben constituir un eje transversal en la formación de los futuros intérpretes. El mejor medio para desarrollar las competencias cultural e intercultural que, según la normativa consultada, forman parte de los perfiles y las competencias de traductores e intérpretes, resulta ser el conocimiento, los conocimientos acerca de las otras culturas que nos permitan sobrepasar los estereotipos y el «reconocimiento de la diferencia sin transformarla en un fetiche identitario» (Camps, 2008, p. XX).

Para ello, disponemos de una poderosa herramienta, Internet, que facilita el acceso a todo tipo de conocimientos culturales en un tiempo récord y permite acercarse a innumerables referentes culturales. Al igual que «en este campo, la televisión fue [...] una fuerza mundialmente esclarecedora» (Newmark, 1999, p. 137), la evolución tecnológica continuará proporcionándonos innovadores medios de comunicación y nuevas TIC que seguirán revolucionando nuestro acceso a las culturas, al igual que los procesos de enseñanza-aprendizaje y, en consecuencia, los roles de estudiantes y docentes.

En cualquier caso, la actitud de estos últimos constituye la pieza clave para favorecer la concienciación de los estudiantes en materia de interculturalidad, intentando destacar siempre las posibles implicaciones culturales de todo contenido tratado, a pesar de que, en ocasiones, nuestros propios conocimientos presenten carencias.

Por último, la integración de elementos culturales en la didáctica de la interpretación permite mejorar la competencia cultural de los alumnos y sensibilizarlos acerca de la diversidad cultural. Con ello se facilita que tomen conciencia y conozcan las diferencias culturales, mejorando entonces su competencia intercultural según la concepción de Witte (2005, p. 50). En definitiva, se trata de que la identidad cultural de los aprendices se vaya desplazando hacia un solapamiento de culturas, hacia una zona fronteriza (Pym, 2003, p. 457). Se trata de formar mentes interculturales, sensibilizadas con ese importante y con frecuencia descuidado aspecto de su futura profesión, mentes capaces de «reconocer y aceptar los logros culturales a los que hace referencia el texto de la lengua origen [...] y, luego, respetar todos los países extranjeros y sus respectivas culturas» (Newmark, 1999, p. 136). Sería posible así sobrepasar discrepancias interculturales que se viven en primera línea en interpretación bilateral y que, sólo a priori, parecen insalvables. 


\section{About the author}

Agustín Darias Marerro es profesor de la Facultad de Traducción e Interpretación de la Universidad de Las Palmas de Gran Canaria.

\section{Article history}

Paper received: $28^{\text {th }}$ October 2015

Paper received in revised form and accepted for publication: $27^{\text {th }}$ September 2016

\section{Referencias bibliográficas}

Agencia Nacional de Evaluación de la Calidad y Acreditación (2004). Libro Blanco del Título de Grado en Traducción e Interpretación. [consulta en línea: http://www.aneca.es/var/media/150288/libroblanco_traduc_def.pdf]

Bowen, M., Bowen, D., Kaufmann, F. y Kurz, I. (1995). «Interpreters and the Making of History» en Delisle, J. y J. Woodsworth (eds.), Translators through History. Amsterdam / Philadelphia, John Benjamins / Unesco, 245-277.

Camps, Assumpta (2008). «Lo uno y lo diverso: traducibilidad y mediación cultural en tiempos globales» en A. Camps y L. Zybatow (eds.), Traducción e interculturalidad. Actas de la Conferencia Internacional «Traducción e intercambio cultural en la época de la globalización», mayo de 2006, Universidad de Barcelona. Frankfurt am Main, Peter Lang, XVII-XXIX.

Collados Aís, Ángela y María Manuela Fernández Sánchez (coord.) (2001). Manual de interpretación bilateral. Granada, Comares.

Collados Aís, Ángela (2004). «La dimensión intercultural en la formación de intérpretes de enlace» en J. A. Gallegos Rosillo y H. Benz Busch (eds.), Traducción y cultura. El papel de la cultura en la comprensión del texto original. Málaga, Libros ENCASA, 139-158.

Corredor Plaja, Anna-María (2011). «Traducción y uso de notas a pie de página: reflexiones a partir de la propia práctica» en M. Sanz y J. Verdegal (eds.), Construcción de identidades y cultura del debate en los estudios en lengua francesa. Castellón de la Plana, Publicacions de la Universitá Jaume I, 150-164.

Darias-Marrero, Agustín y Jessica Pérez-Luzardo Díaz (2003). «Competencias comunes para la formación en interpretación consecutiva y simultánea» en A. Collados Aís, M. M. Fernández Sánchez, E. M. Prada Macías, C. Sánchez Adam y E. Stévaux (eds.), La evaluación de la calidad en interpretación: docencia y profesión. Granada, Comares, 155-162. 
Darias-Marrero, Agustín (2009). «La situación comunicativa y sus constituyentes en interpretación» en S. Bravo Utrera y R. García López (eds.), Estudios de traducción: Perspectivas. Frankfurt am Main, Peter Lang, 32-49.

Darias-Marrero, Agustín, Soraya García Sánchez y Ana Ruth Vidal Luengo (2013). «Aprendizaje móvil, ubicuo y autónomo de lenguas extranjeras en la ULPGC» en E. Llavero Ruiz (coord.), Cuadernos de innovación educativa 1. Las Palmas de Gran Canaria, Servicio de Publicaciones y Difusión Científica de la ULPGC, 11-35.

Félix Fernández, Leandro (2004). «El análisis de la situación de comunicación como medio para averiguar la intencionalidad del emisor. Un ejemplo ilustrativo» en J. A. Gallegos Rosillo y H. Benz Busch (eds.), Traducción y cultura. El papel de la cultura en la comprensión del texto original. Málaga, Libros ENCASA, 21-53.

García López, Rosario (2004). Guía didáctica de la traducción de textos idiolectales. La Coruña, Netbiblo.

Gile, Daniel (1984). «Les noms propres en interprétation simultanée». Multilingua, 3-2, 79-86.

Gile, Daniel (1985). «Le modèle d'efforts et l'équilibre d'interprétation en interprétation simultanée». Meta, 30-1, 44-48.

Gile, Daniel (1988). «Le partage de l'attention et le modèle d'efforts en interprétation simultanée». The Interpreters' Newsletter, 1, 4-22.

Gile, Daniel (2004). «L'interprétation de conférence et la temporalité». file:///D:/LFE/RISC, \%20cours\%20Ecole,\%20Interpr\%C3\%A9tation\%20de\%20conf\% C3\%A9rence\%20et\%20temporalit\%C3\%A9.htm [acceso: 21-09-16].

Göhring, Heinz (1978). «Interkulturelle Kommunikation: Die Überwindung der Trennung von Fremdsprachen- und Landeskundeunterricht durch einen integrierten Fremdverhaltensunterricht» en M. Hartig y H. Wode (eds.), Kongressberichte der 8. Jahrestagung der Gesellschaft für Angewandte Linguistik (GAL) e.V., Mainz 1977. Stuttgart, Hochschulverlag, vol. 4, 9-14.

Goodenough, Ward H (1964). «Cultural Anthropology and Linguistics» en D. Hymes (ed.), Language in Culture and Society: A Reader in Linguistics and Anthropology. New York, Harper \& Row, 36-40.

Gumul, Ewa (2008). «Explicitation in simultaneous interpreting. The quest for optimal relevance» en E. Walaszewska, M. Kisielewska-Krysiuk, A. Korzeniowska y M. Grzegorzewska (eds.), Relevant Worlds: Current Perspectives on Language, Translation and Relevance Theory. Newcastle upon Tyne, Cambridge Scholars Publishing, 188-205.

Hurtado Albir, Amparo (1999). Enseñar a traducir. Metodología en la formación de traductores e intérpretes. Madrid, Edelsa Grupo Didascalia.

Koskinen, Kaisa (2004). «Shared culture? Reflections on recent trends in Translation Studies». Target, 16-1, 143-156.

Kottak, Conrad Phillip (1994). Antropología. Una exploración de la diversidad humana. Madrid, Mac Graw Hill. 
Kroeber, Alfred Louis (1948). Anthropology: race, language, culture, psychology, pre-history. New York, Harcourt, Brace and company.

Kurz, Ingrid (2003). «Physiological stress during simultaneous interpreting: A comparison of experts and novices». The Interpreters' Newsletter, 12, 51-67.

Ladmiral, Jean-René (1998). «Le prisme interculturel de la traduction». Palimpsestes, 11, 15-30.

Lvóvskaya, Zinaida (1997). Problemas actuales de la traducción. Granada, Método Granada Lingüística.

Marín Hernández, David (2005). «La esencialización de la cultura y sus consecuencias en los estudios de traducción». TRANS, 9, 73-84.

Martínez Sahuquillo, Irene (1997). «Los dos conceptos de cultura: entre la oposición y la confusión». REIS, 79, 173-196.

Ministerio de Educación, Cultura y Deporte (2002). Marco común europeo de referencia para las lenguas: aprendizaje, enseñanza, evaluación. Madrid, Secretaría General Técnica del MECD, Subdirección General de Información y Publicaciones, y Grupo ANAYA, S.A.

Muñoz Martín, Ricardo (1999). «Contra Sísifo: Interdisciplinariedad y multiculturalidad». Perspectives: Studies in Translatology, 7-2, 153-163.

Newmark, Peter (1999). Manual de traducción. Madrid, Cátedra.

Nord, Christiane (1997). Translating as a Purposeful Activity. Functionalist Approaches Explained. Manchester, St Jerome Publishing.

Nord, Christiane (2008). La traduction : une activité ciblée. Introduction aux approches fonctionnalistes. Arras, Artois Presses Université.

Pacte (2003). «Building a Translation Competence Model» en F. Alves (ed.), Triangulating Translation: Perspectives in process oriented research. Amsterdam, John Benjamins, 43-66.

Pacte (2014). «First Results of PACTE Group's Experimental Research on Translation Competence Acquisition: The Acquisition of Declarative Knowledge of Translation». MonTI, núm. especial 1, 85-115.

Plog, Fred y Daniel G. Bates (1980). Cultural Anthropology. New York, Alfred A. Knopf.

Pöchhacker, Franz (2003). «Enfoque funcional de la interpretación simultánea» en A. Collados Aís y J. A. Sabio Pinilla (eds.), Avances en la investigación sobre interpretación. Granada, Comares, 105-122.

Pym, Anthony (2003). «Alternatives to Borders in Translation Theory» en S. Petrilli (ed.), Translation Translation. Amsterdam y New York, Rodopi, 451-463.

Rocher, Guy (1969). Introduction générale à la sociologie. Regards sur la réalité sociale. Montréal, Hurtubise $\mathrm{HMH}$, vol 1.

Tylor, Edward Burnett (1871). Primitive Cultures: Researches into the Development of Mythology, Philosophy, Religion, Language, Art and Custom. London, John Murray, vol. 2.

Vermeer, Hans J. (1986). Voraus-setzungen für eine translationstheorie. Einige kapitel kultur- 
und sprachtheorie. Heidelberg, Vermeer.

Vermeer, Hans J. (1992). Skopos und Translationsauftrag - Aufsätze. Frankfurt am Main, Verlag für Interkulturelle Kommunikation.

Williams, Raymond (1983). Keywords. A vocabulary for culture and society. New York, Oxford University Press.

Witte, Heidrun (2000). Die Kulturkompetenz des Translators: Begriffliche Grundlegung und Didaktisierung. Tübingen, Stauffenburg-Verlag.

Witte, Heidrun (2005). «Traducir entre culturas. La competencia cultural como componente integrador del perfil experto del traductor». SENDEBAR, 16, 27-58.

Witte, Heidrun (2008). Traducción y percepción intercultural. Granada, Comares. 